\begin{tabular}{ccc}
\hline International Journal of Engineering \& Technology, $7(4.5)(2018) 542-547$ \\
SPC & Website: $\frac{w w w . s c i e n c e p u b c o . c o m / i n d e x . p h p / I J E T}{2}$ \\
Research paper & Technology \\
\hline
\end{tabular}

\title{
Findings of performance evaluation of EDM for different materials of electrodes and work pieces- a review
}

\author{
Harshalkumar R. Mundane ${ }^{1 *}$, Dr. A. V. Kale ${ }^{1}$, Dr. J. P. Giri ${ }^{1}$ \\ ${ }^{1}$ Department of Mechanical Engineering, Yeshwantrao Chavan College of engineering, Nagpur - 441110, India \\ *Corresponding author E-mail: harshalmundane1@gmail.com
}

\begin{abstract}
EDM (Spark erosion) is non-conventional machining process which uses as removing unwanted material by electrical spark erosion. EDM Machining parameters affecting to the performance and the industries goal is to produce high quality of product with less time consuming and cost. To achieve these goals, optimizing the machining parameters such as pulse on time, pulse off time, cutting speed, depth of cut, duty cycle, arc gap, voltage etc. The performance measure of EDM is calculated on the basis of Material Remove Rate(MRR), Tool Wear Rate(TWR), and Surface Roughness(SR).

The main objective of present work is to investigate of the influence of input EDM (Electro Discharge Machining) parameters on machining characteristics like surface roughness and the effects of various EDM process parameters such as pulse on time, pulse off time, servo voltage, peak current, dielectric flow rate, on different process response parameters such as material removal rate (MRR), surface roughness (Ra), Kerf (width of Cut), tool wear ratio(TWR)and surface integrity factors. In this paper few selected research paper related to Die-sinker EDM with effect of MRR, TWR, surface roughness (SR) and work piece material have been discussed.
\end{abstract}

Keywords: Electro Discharge Machining (EDM); Dielectric; Process Parameters; Performance Parameters; Material Removal Rate(MRR);Tool Wear Rate (TWR); Surface Roughness (SR).

\section{Introduction}

Electrical discharge machining (EDM), also known as spark machining, spark eroding, burning, die sinking, wire burning or wire erosion. Electrical Discharge Machining (EDM) is a modern manufacturing process machining process, where electrically con- ductive material is removed by controlled erosion through a series of electric sparks of short duration and high current density be- tween the electrode and the work-piece were both are submerged in a dielectric bath, containing kerosene or distilled water. During this process thousands of sparks per second are generated, and each spark produces a tiny crater in the material along the cutting path by melting and vaporization. Generally the material is re- moved by erosion process. The top surface of the work-piece sub- sequently re-solidifies and cools at a very high rate. The applica- tion of this process is mostly found in press tools and dies, plastic moulds, forging dies, die castings, aerospace, automotive, surgical components manufacturing industries etc. This process is not re- stricted by the physical and metallurgical properties of the work material as there is no physical contact due to high-energy electro- thermal erosion between the tool and the work-piece.

It uses electro-thermal phenomenon, coupled with surface ir- regularities of the electrodes, interactions between two succes- sivedischarges and presence of debris particles makes the EDM process too abstruse, so that complete and accurate physical mod- elling of the process has been observed to be difficult to establish. The favorable EDM process parameters selection is required for obtaining the best machining performance by increasing the pro- duction rate at the same time reducing the machining time. The process parameters are generally determined based on experience or on handbook values. However, this does not confirm that the chosen machining parameters result in optimal or near optimal machining performance of the EDM process.

\subsection{History of EDM}

Russian scientist Lazarenko studied the electric dis- charge for metal cutting application and developed spark erosion machine in 1943. EDM process has been in use since 1952 English scientist Priestly studied in detail the erosive action of electric discharges on metals in 1970. Ero- sion of metals by spark was first reported by Joseph Priest- ley in 1978 and electric-sparking has been utilized since long for its by- product, "colloidal metal powder", but sparks were not used for machining as much until the late thirties when the tap disintegrator was introduced. However, controlled machining by electric sparks was first introduced by Lazarenko in Russia in 1944. The first British patent was granted to Rudorff in 1950. USA, Japan and Switzerland developed their machines around 1950. A machine for spark machining by 'method $\mathrm{x}$ ' was patented in USA in 1952 .

\subsection{Working principle}

In the principle of working of EDM is based on the erosion of the material by repetitive sparks between the work-piece and the tool submerged in a bath of the dielec-tric medium. The work-piece and are two tools are two electrodes connected by D.C. pulse generator. Electrodetool is shaped tool of required profile to be cut on the work- piece. The work-piece and electrode are separated by a gap to establish a pulsated spark across which the dielectric fluid flows. The servo drive system maintains the spark gap for continuous operation with desirable feed of the tool. 


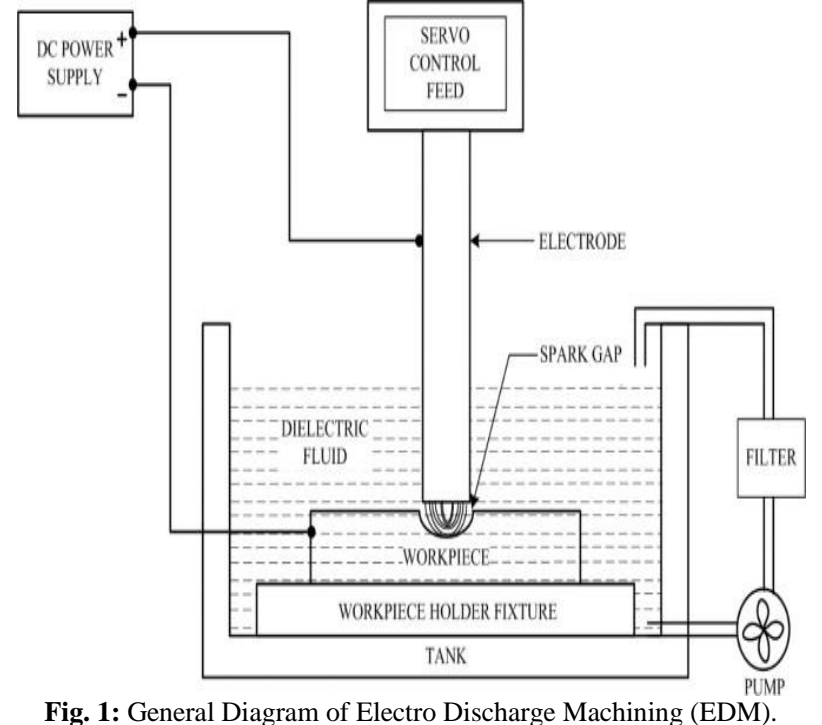

Fig. 1: General Diagram of Electro Discharge Machining (EDM).

\section{Types of EDM}

There are two different types of EDM:

1) Die-sinking

2) wire-cut

\subsection{Die-sinking EDM}

Sinker EDM machine use an electrode and work piece submerged in liquid as oil or dielectric fluid. In the Die sink EDM, two metal parts submerged in an insulating liquid are connected to a source of current which is switched on and off automatically depending on the parameters set on the controller. When the current is switched on, an electric ten- sion is created between the two metal parts. If the two parts are brought together to within a fraction of an inch, the electrical tension is discharged and a spark jumps across. Where it strikes, the metal is heated up so much that it melts. Sinker EDM, also called cavity type EDM or volume EDM consists of an electrode and work piece submerged in an insulating liquid such as, more typically, oil or, less fre- quently, other dielectric fluids. The electrode and work piece are connected to a suitable power supply. The power supply generates an electrical potential between the two parts. As the electrode approaches the work piece, dielectric breakdown occurs in the fluid, forming a plasma channel, and a small spark jumps.

\subsection{Wire-cut EDM}

Eire EDM uses an electrode and spark to cut metal. EDM sometimes called travelling wire EDM. Electrical discharge wire cutting is a process that is similar in config- uration to band swing except in the case of WEDM the saw is a wire electrode of small diameter. Material removal is affected as a result of spark erosion as the wire electrode is fed through the work piece. In most cases, horizontal movement of the worktable, is controlled by CNC.

Wire-cutting EDM is commonly used when low resid- ual stresses are desired, because it does not require high cutting forces for removal of material. If the energy/power per pulse is relatively low (as in finishing operations), little change in the mechanical properties of a material is ex- pected due to these low residual stresses, although material that hasn't been stress-relieved can distort in the machining process. Due to the inherent properties of the process, wire EDM can easily machine complex parts and precision components out of hard conductive materials.

\section{Parameters of EDM}

\subsection{Process parameters of EDM}

1) Spark on time (pulse time or Ton):- It is the dura- tion of time for which current is allowed to flow per cycle. Material removal is directly proportional to the amount of energy applied during this on- time. It is the duration of time expressed in micro seconds.

2) Spark Off-time (pause time or Toff):-The dura- tion of time ( $\mu \mathrm{sec})$ between the sparks (that is to say, on-time). This time allows the molten material to solidify and to be wash out of the arc gap. This parameter is to affect the speed and the stability of the cut. Thus, if the off-time is too short, it will cause sparks to be unstable.

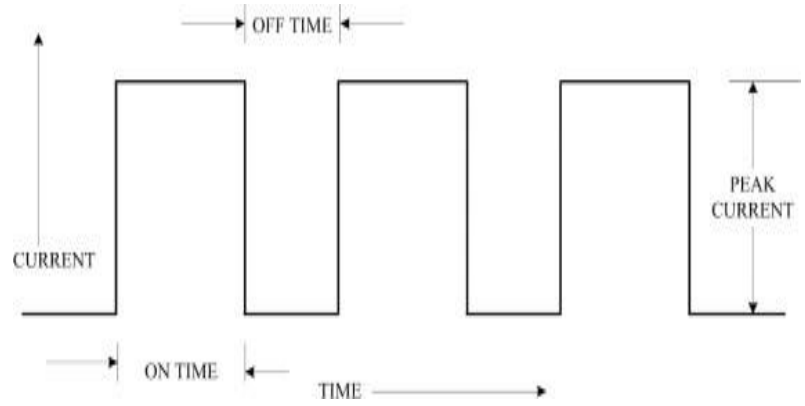

Fig. 2: Pulse Waveform of Pulse Generator

3) Pulse Frequency: Pulse Frequency is defined as number of cycles produced at the gap in one second.

Pulse Frequency (KHz)

$=1000 /($ Total Cycle Time $(\mu \mathrm{sec}))$

$=1000 /($ Pulse on + Pulse off $(\mu \mathrm{sec}))$

4) Polarity:-The Polarity normally used is normal polarity in which the tool is negative and work piece is positive. Sometimes positive polarity can be used depending upon the requirement, where tool is positive and work piece is negative. The negative polarity of the work piece has an inferior surface roughness than that under positive polarity in EDM. MRR is higher when tool is connected to positive polarity.

5) Arc Gap: The arc gap is the distance between the electrode and work piece during the process of EDM. It may called as spark gap. Spark gap can be maintained by servo system. It is very thin gap in the range of $10-125 \mu \mathrm{m}$. An electro-mechanical and hydraulic systems are used to respond to average gap voltage. To obtain good performance and gap stability a suitable gap should be maintained. For the reaction speed, it must obtain a high speed so that it can respond to short circuits or even open gap circuits. Gap width is not measured directly, but can be inferred from the average gap voltage.

6) Discharge Current (IP):- Current is measured in ampere (A). Discharge current is responsible di- rectly for material removal. It contains energy for melting and evaporation.

7) Duty Cycle $(\tau)$ :- It is a percentage of the on-time relative to the total cycle time. This parameter iscalculated by dividing the on-time by the total cy-cle time (on-timepulse off-time). The result is mul- tiplied by 100 for the percentage of efficiency or the so-called duty cycle.

Duty cycle $=\frac{T_{O N}}{T_{O N}+T_{O F F}}$

8) Voltage (V):- It is a potential that can be measure by volt it is also effect to the material removal rate and allowed to per cycle.

9) Intensity: It points out the different levels of power that can be supplied by the generator of the EDM machine.

10) Over cut: It is the clearance per side of the elec- trode and the work piece after the machining oper- ation. It is the measure 
of cut produced exceeding the diameter of the tool. The impression created while EDM process is generally slightly larger than the original diameter of the tool electrode. This is because the spark is generated from along the side of the tool and hence erosion takes place in that direction also. OC is calculated as half the difference of the diameter of the hole produced to the tool diameter.

\subsection{Performance parameters of EDM}

1) Material Removal Rate (MRR):- MRR is the rate at which the material is removed the work- piece. Electric sparks are produced between the- tool and the workpiece during the machining pro- cess. Each spark produces a tiny crater and thus erosion of material is caused. The MRR is defined as the ratio of the difference in weight of the workpiece before and after machining to the densi- ty of the material and the machining time.

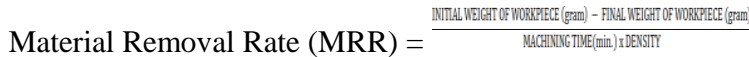

2) Tool Wear Rate (TWR):-TWR is a performance measure for the erosion rate of the tool considering the geometrical accuracy of the machined feature. The TWR is defined as the ratio of the difference in weight of the tool before and after machining to the machining time.

Tool Wear Rate $($ TWR $)=\frac{\text { INITALWEIGHT OF TOOL (gram) - FINAL WEICHT OF TOOL (gram) }}{\text { MACHINIG TIME(min.)x DENSTYY }}$

3) Surface Roughness: Roughness is a measure of the texture of a surface. It is quantified by the ver- tical deviations of a real surface from its ideal form. If these deviations are large, the surface is rough; if they are small the surface is smooth. Roughness is typically considered to be the high frequency, short wavelength component of a measured surface. Surface roughness isan im- portant measure of product quality since it greatly influences the performance of mechanical parts as well as production cost. Surface roughness has an impact on the mechanical properties like fatigue.

Behavior, corrosion resistance, creep life, etc. sur- face roughness tester to measure surface roughness of work piece.

\section{Work piece \& tool material use in EDM}

\subsection{Work piece materials}

There are different types of work piece material are using the EDM method. It is capable of machining geometrically complex or hard material components, that are precise and difficult-to- machine such as heat treated tool steels, composites, super alloys, ceramics, carbides, heat resistant steels etc. List of Workpiece which are used in EDM : EN31 Tool Steel, Tungstan-Carbied, V Composite, Al 7075 B4C MMC, AISI 202 SS, AISI D3 Tool Steel, H-11 Steel, H-13 Tool Steel, Hastelloy Steel, Mild Steel, AISI 1040 Medium Carbon Steel, EN19, EN9, AISI 316-L SS, NiTi60-SMA, AISI D2 Tool Steel, Ai-SiCP MMC, AISI P20 Tool Steel, Silver Steel, W300 Die Steel, AISI 4340 Steel, Titanium Super Alloy.

\subsection{Tool material}

Tool material should be such that it would not undergo much tool wear when it is impinged by positive ions. Thus the localized temperature rise has to be less by tailoring or properly choosing its properties or even when temperature increases, there would be less melting.

\subsubsection{The characteristic of EDM tool are}

1) High electrical conductivity
2) High thermal conductivity

3) High melting temperature

4) Higher Density

5) Cheapness

\subsubsection{List of tool material which are used in EDM}

1) Copper: Copper is good conductor of heat and electricity. It is a soft, malleable and ductile metal. Copper as the primary electrode material, due to their tool making culture that is averse to the "unti- diness" of working with graphite. Due to its structur- al integrity, Copper can produce very fine surface finishes, even without special polishing circuits. This same structural integrity also makes Copper elec- trodes highly resistant to DC arcing in poor flushing situations. Copper is frequently used in reverse burn- ing punches and cores in the Sinker EDM. Most of researchers used this material for the machining of work piece.

2) Graphite:-Graphite is a naturally-occurring form of crystalline carbon. Graphite is an excellent conduc- tor of heat and electricity and has the highest natural strength and stiffness of any material. It maintains its strength and stability to temperatures in excess of 3600c. It is extremely soft, cleaves with very light pressure, and has a very low specific gravity. Graph- ite is the preferred electrode material for $95 \%$ of all sinker EDM applications. Thus, it is important that we expend considerable effort to understand its properties and application to EDM

3) Brass:-Brass is a metallic alloy that is made of cop- per and zinc. Brass is used for decoration for its bright gold like appearance The applications where low friction is required such as locks, gears, bearings, doorknobs, ammunition castings and valves. Brass is one of the first EDM electrode materials. It is eco- nomical and easy to machine. Today, brass is less used as an electrode material in modern sinker EDMs, due to its high wear rate.

4) Silver:-Silver is the highest electrical conductivity, thermal conductivity and reflectivity of any metal. Silver is occasionally used as an electrode material, due to its superior purity, and structural integrity. The use of Silver electrodes and fine finish can pro- duce extraordinary fine finishes in coining dies, where the use of orbiting to improve the finish would distort the cavity detail. Most silver is pro- duced as a byproduct of copper, gold, lead, and zinc refining.

5) Tungsten:-Tungsten is a hard steel- grey metal that is often brittle and hard to work. Tungsten has the highest melting point, lower vapor pressure, and highest tensile strength. Due to the combination of its high density, tensile strength, and melting point, Tungsten had been the electrode material of choice for certain limited EDM applications. It is important to note that Tungsten, due to its relatively poor elec- trical conductivity, cuts much slower than Brass or Copper. Also, due to its high cost and very low ma- chinability, Tungsten is seldom used.

6) Copper Tungsten:-Commonly used copper tungsten mixtures contains $10-50 \mathrm{wt}$ \% of copper, the remain- ing portion being mostly tungsten. Copper Tungsten $(\mathrm{CuW})$ is a powder metal product designed to com- bine the best EDM properties of Copper and Tung- sten. Copper Tungsten combines the high electrical conductivity of copper with the high melting point of tungsten. The combination of these two metalscre- ates an electrode material with very good wear prop- erties. Copper Tungsten is unmatched for its wear resistance, holds up very well in sharp corners, and is readily machined and ground without the burr is- sues associated with Copper.

7) Silver Tungsten:-The high thermal and electrical conductivities of silver with the arc resistance of tungsten. Silver Tungsten is a powder metal product which combines the wear resistance of Tungsten with the high conductivity of Silver, to give an un- matched combination of low wear and fine surface finish for EDM applications with fine detail. Silver 
Tungsten is made by the same process as Copper Tungsen. Due to its high cost and limited availability, Silver Tungsten has a very limited range of applica- tions. They are used in heavy duty devices subject to high currents.

8) Tungsten Carbide: Tungsten carbide is a fine grey powder, but it can be pressed and formed into shapes for use in industrial machinery, cutting tools, abra- sives, armor-piercing round, other tools and instru- ments and jewelry. Tungsten carbide is extremely hard and low electrical resistivity Due to its extraor- dinary stiffness and low wear properties, Tungsten Carbide is often the preferred electrode material.

9) Aluminum:-Aluminum is a relatively soft, durable, lightweight, ductile, and malleable metal. It is Easily available \& Economical. Aluminum is most widely used in non-ferrous metal.

\section{Literature review}

Senthil Kannan et al. (2017) selected the machining of AL 2017 alloy as workpiece materials and electrode mate- rials as copper, Brass, and Silver. They have taken tree pa- rameters, namely; pulse on, pulse off, current to observed the Material removal Rate (MRR),Tool wear Rate(TWR), and Surface roughness(SR). The experiments were con- ducted by Taguchi method with L27 orthogonal table. The ANOVA analysis is utilized for finding the influence of each variable and optimal processing parameter and verified through experimentation to improve process. They suggest that suggested optimal machining parameters for obtaining the maximum MRR with less TWR and Surface Roughness [1]. Satish Kumar et al. (2017) used as powder material in dielectric fluid to improve Material Remove Rate (MRR), Tool Wear Rate (TWR), and surface roughness. They are used for machining different powder particles in dielectric fluid in EDM such as aluminum powder, silicon carbide, graphite etc. They used Inconel 800 as workpiece material and copper, copper-chromium and graphite as electrode materials. They are used Different input parame- ters such as peak current, pulse on-time, pulse off-time. They show that peak current, pulse on-time, and tool mate- rial significantly affects the Material Removal Rate (MRR) and peak current, pulse on-time, tool material and powder materials affected the Tool Wear Rate (TWR). Pulse off- time has a trifling effect on both MRR and TWR, while powder particles on MRR [2].Trinkle Yuvaraj Saindane et al. (2016) is used Taguchi method for solving the specific problem. They are used to conduct experiments, L27 Taguchi orthogonal array (OA). They are used workpiece material as AISI H13 tool steel and electrode material as copper. They are take Process parameters as Discharge current, Spark On-time, Spark Off-time, Dielectric level, Flushing Pressure. Applying ANOVA, the effect of each input parameter on SR can be determined. They are used Taguchi method for the application of in the analysis of experimental result. They conclude that the surface rough- ness increases with the increase in discharge current and surface roughness decreases with the increase of pulse on- time and pulse off-time. They also conclude that Taguchi's robust Orthogonal Array design method is suitable to ana- lyze the surface roughness problem solving [3].

N. Mathan Kumar et al.(2016) investigated the influ- ence of process parameters and their interactions viz., Tem- perature, pulse on time, pulse off time and current on the Material Removal Rate (MRR) in Aluminium 2618 alloy as workpiece. They are use copper as electrode material It used to analyze the effect of the parameters on Material Removal Rate (MRR), Tool wear rate (TWR), Surface roughness (SR). Parameters contribution was calculated by the Analysis of Variance (ANOVA). The input and output parameters were examined with help of Genetic Algorithm (GA). They are concluded that the minimum Tool Wear Rate (TWR), Material Removal Rate (MRR) and Depth

were identified in higher Wt. \% of Al 2618 composite[4]. Vijaykumar S. Jatti et al. (2016) used Taguchi methodology to optimize EDM process parameters for the machining of NiTi alloy, $\mathrm{NiCu}$ alloy and $\mathrm{BeCu}$ alloy. They used copper as electrode material. They have taken four EDM parame- ters, namely; Current, Gap Voltage Pulse on time, Pulse off time and to observe Tool Wear Rate(TWR). Experiments were carried out as per the Taguchi's L18 orthogonal array. They concluded that electrical conductivity, gap current and pulse on time are significant process parameters that influ- ence tool wear rate [5]. D. Vinoth kumar et al. (2016) pre- sented an Investigation of effect and optimization of ma- chining parameters namely; pulse on time, pulse off time, Current on Material Removal Rate(MRR) and Tool Wear Rate(TWR). L9 Mixed orthogonal Array is used for exper- imentation. Incoloy 600 is used as workpiece material and Copper is used as electrode material. They are taken Opti- mization using Taguchi-Grey RelationalAnalysis. The experimental results are validated with ANOVA Method. They concluded that process parameters more influence on Material Removal Rate (MRR) [6].

WS Wan Harun et al. (2016) is used Taguchi Method for experimental analysis. They are used workpiece materi- al as mild steel (AS3679) and electrode material as copper. Three drilling parameters such as pulse off time, peak cur- rent and servo standard voltage and performance parame- ters are Material Removal Rate (MRR). They are used the Analysis of Variance (ANOVA) test is conducted. The ex- perimental table is setting up using L9 orthogonal array. They are used Taguchi Method for modeling. They conclude that the peak current and standard voltage are the main parameters from three controllable factors, based on the percentage of contribution [7]. Mr. Somnath M. Kale et al. (2016) had analyzed optimization of process parameters for machining of Inconel 718. They are taken three Voltage, Current, Pulse on time for observation of Material Removal Rate (MRR), and Tool Wear Rate (TWR). They are used workpiece material as Inconel 718 and electrode material as Brass. They are used ANOVA techniques are used for data analysis. They are conclude that Current is most affecting parameters for both MRR and TWR. Material removal rate and tool wear rate increases as the current and pulse on time increases. Material removal rate and tool wear rate decreas- es as the voltage increases [8]. Balbir Singh et al.(2015)is used tungsten powder in dielectric fluid for improving per- formance. He take DM for experimentation and optimum parameters as: peak current, pulse on time, pulse off time, gap voltage they take AA6061 Material for machining. By the use of powder they conclude that surface hardness is increased when machining with powder mixed in EDM [9]. Rupesh Kumar Tiwari et al. (2015) used experimental analysis for genetic algorithm and Multi-objective optimi- zation parameters such as Discharge Current (A), Pulse on time, and Pulse off time of electrical discharge machine. They are observed machine of Material remove rate (MRR) and surface finish.They chose the work piece material as Ti 6-4 and the tool or electrode material as copper. They conclude that The MRR is increasing with increase in dis- charge current almost linearly. Pulse on time initially at slower rate but later the increase is at a faster rate, The MRR is decreasing with increase in pulse off time almost linearly [10]. V.Balasubramaniam et al. (2014) used for ex- perimental analysis artificial neural network They take dif- ferent electrode materials namely copper, brass and tung-sten and workpiece materials are Al-SiCp Metal. The per- formance parameters are Material Removal Rate (MRR), Electrode Wear Rate (EWR), and Circularity (CIR) are considered. The input parameters are current, pulse on time, pulse off time. They conclude that the ANN model is poten- tial to predict the neural network architecture [11]. Kapil Banker et al. (2014) used optimum Parameters of Electro Discharge Machine. He use experimental analysis using Taguchi Method. He used workpiece material as AISI 304 Steel and AISI 304L stainless steel and electrode material as copper material he used optimum parameters Current, Pulse on time, Pulse of time and on machining observe MRR. They are used using L9 orthogonal array for experi- mental process. Using TaguchiMethod for experimental analysis. They are finding that the copper having high ma- terial removal rate with respect to other material such as aluminum, gun metal, brass, etc.[12].

Dr. M.Indira Rani et al. (2014), they used Optimization of Various Machining Parametersof Electrical Discharge Machining (EDM) Process They used workpiece material as AISI D2 Tool Steel and 
electrode material as Copper. They used experimental analysis Using Hybrid Optimiza- tion Method and used optimum parameters are Discharge current, Pulse on time (Ton), Duty cycle (Tau), Voltage and observe machine process for Material Removal Rate(MRR) and EWR. Taguchi L9 array with grey relational analysis has been used to optimize the multiple performance charac- teristics such as material removal rate (MRR) and Electrode wear rate (TWR). They are concluded that increase in pulse ON time, the value of MRR first increased and then gradu- ally decreased after level-2 (200 $\mu \mathrm{sec})$ but the value of EWR gradually decreased from level-1(100 $\mu \mathrm{sec})$ to level-4 $(75 \mu \mathrm{sec})$ [13]. Chandramouli set et al. (2014) analysis op- timize process parameters on machining of RENE80 Nickel Super alloy. They have taken three EDM parameters namely current, pulse on time, pulse off time and machining du- ration to observed the Material Remove Rate(MRR), Tool Wear Rate(TWR), Surface Roughness(SR). They are used Taguchi method for formulate the experimental layout and ANOVA method is used to analysis the effect of input pro- cess parameters. They conclude that proper selection of input parameters will play a significant role in Electric Dis- charge Machining [14]. S. Raghuraman et al.(2013) is used optimization of EDM parameters like process parameters such as current, pulse ON and OFF time in Electrical Dis- charge Machining (EDM) identify the variations in material removal rate (MRR), Tool wear rate(TWR), and surface roughness value. They work material for machining Mild Steel IS 2026 used and for electrode material copper as used. They conduct experimentation with the help of L9 orthogonal array. For the experimental analysis they used optimization using grey relational analysis taguchi meth- od.The conclusion explain that the taguchi grey relational analysis is being effective technique to optimize the ma- chining [15].

M.A. Ali et al. (2013) analysis experimentally by the use of ANOVA method. They explain Effect of EDM Die- sinking Parameters on Material Removal Rate. They are used Beryllium Copper $(\mathrm{BeCu})$ as workpiece material and copper as electrode material Peak Current (A), Machine Voltage (V), Pulse on Time ( $\mu$ sec), Pulse off Time ( $\mu \mathrm{sec})$ used as optimum process parameters. They conclude that changes in peak current, pulse on time have contributed to influence of MRR. [16]. S. Assarzadeh et al. (2013) used optimum process parameters like discharge current, pulse on time, pulse off time, duty cycle and observe on machin- ing surface roughness They experimentally analysis con- duct with the help of ANOVA method. He suggest that MRR increase by selecting both higher discharge current and duty cycle and they suggest that for lower TWR, longer pulse on time with lower current while smooth work sur- face [17]. M. Gostimirovic et al. (2012) explain effect of electrical pulse parameters on machining performance in EDM. They used Discharge current and pulse duration, gap distance as the electrical pulse parameters. Observe ma- chining influence on material remove rate, Tool wear rate, and surface roughness. They are used graphite as a tool material for experimentation. They conclude that machining performance of EDM directly depends on the electrical pulse parameters and most important parameters are discharge current and pulse duration. When electric pulse pa- rameters are increased, the gap distance has a greater influ- ence on the machining accuracy [18].

Ramezan Ali MahdaviNejad et al. (2011) used experi- mental analysis by Artificial Neural Network and Non- dominating Sorting Genetic Algorithm (NSGA II). ANN for model the process and GA used for optimize the process. Silicon Carbide as workpiece material and electrode mate- rial as Copper. Input process parameters are discharge cur- rent, pulse on time (Ton), and pulse off time (Toff) on elec- tric discharge machining. In experimentation An ANN model has been trained within the experimental data. Vari- ous ANN architectures have been studied [19].P. Kuppan et al. (2008)experimental analysis of small deep hole drilling in EDM. They used workpiece material as Inconel 718 and electrode material as copper in EDM process. The parame- ters take such as peak current, pulse on-time, duty factor and observe machining parameters like material removal rate (MRR) and depth averaged surface roughness (DASR). The experimentation conduct using central composite de- sign (CCD) procedure. They conclude that the MRR in- creases with the increase in peak current, duty factor and electrode speed. The DASR increases with the increase in peak current and pulse on-time. to achieve better surface finish low value of pulse on-time to be selected [20].M. Kiyak et al. (2007), they select workpiece material as 40CrMnNiMo864 (AISI P20) and electrode materials as copper. They used optimum parameters as Pulsed current (A), Pulse on time, Pulse off time. They observed that Sur- face finish quality was better when applying smaller pulsed current and pulse time. High pulsed current and pulse time provide low surface finish quality. They concluded that workpiece surface roughness will be increasing due to wear rate on electrode [21].

Kun Ling Wu et al. (2005) used experimental analysis Taguchi method and aluminium powder, surfactant in the dielectric fluid. For the experimentation used optimum pa- rameters as: discharge current, pulse on time, pulse off time, gap voltage. They used Taguchi L18 orthogonal array was employed to explore the relation between surfactant and machining performance. They conclude that surfactant add- ed in dielectric improve surface roughness of work piece [22].

\section{Conclusions}

It is observed that different types of performance parame- ters are applied by different authors. It is found that different types of optimization techniques are used to optimize the machining parameters in electrical discharge machining (EDM). The performance is affected by discharge current, pulse on time, pulse off time, arc gap, duty cycle, servo voltage. Mostly research work carries out the Material re- moval rate (MRR), Tool wear rate (TWR), Surface roughness $(\mathrm{Ra})$, etc. for different types of work material.

\section{Acknowledgement}

Author honestly acknowledge the infrastructural support from Dr. A.V. Kale, J. P. Giri for the present work. All those who contributed directly and indirectly are thanked.

\section{References}

[1] Senthil Kannan.V, Dr. K. Lenin, "Selection of optimum parameter and Electrode for machining of AL 2017 Alloy by machining in Electrical discharge Machining" International Journal of Mechanical Engineering- (ICEHS), (2017).

[2] Satish Kumar, Ashwani Kumar Dhingra and Sanjeev Kumar, "Parametric optimization of powder mixed electrical dis- charge machining for nickelbased superalloy inconel-800 us- ing response surface methodology" Mechanics of Advanced Materials and Modern Processes, (2017).

[3] Trinkle Yuvaraj Saindane, H. G. Patil, "Optimization of Var- ious Process Parameter of EDM Using Taguchi Method with Experimental Validation" International Journal of Engineer- ing Science and Computing, (2016).

[4] N. Mathan Kumar, S. Senthil Kumaran, L.A. Kumaraswa- midhas "High temperature investigation on EDM process of Al 2618 alloy reinforced with Si3N4, ALN and ZrB2 in-situ composites" Journal of Alloys and Compounds, (2016).

[5] Vijaykumar S. Jatti, T. P.Singh " Optimization of Tool Wear Rate during Electrical Discharge Machining of Advanced Materials using Taguchi Analysis" WSEAS Transactions on Applied and Theoretical Mechanics (2016).

[6] D.Vinoth Kumar, P.Siva Kumar, B.Kumaragurubharan, T.Senthil kumar, "Experimental Investigation of Process Pa- rameters in EDM for Incoloy 600 Using Taguchi-GRA” In- ternational Journal of Engineering Science and Compu- ting, (2016).

[7] MFF Ab. Rashid, WS Wan Harun and SA Che Ghani, "Op- timization of edm small hole drilling process using taguchi approach" Journal of Engineering and Applied Scienc- es,(2016).

[8] Mr. Somnath M. Kale, Mr.D.S.Khedekar, "Optimization of Process parameters in EDM for Machining of Inconel 718 us- ing Response Surface Methodology" International Journal of Innovations in Engineering and Technology (IJIET), (2016). 
[9] Jatinder kumar, Balbir singh, sudhir kumar, "Investigation of the tool wear rate in tungsten powder mixed electric dis- charge machining of AA6061/10\% Sic p composite", Journal of Material and Manufacturing Process, (2015).

[10] Rupesh Kumar Tiwari, "Multi-objective optimization of electrical discharge machining process parameters using genetic algorithm"Journal of Engineering Research and General Sci- ence, (2015).

[11] V.Balasubramaniam, N.Baskar, C.Sathiya Narayanan, "Op- timization of electrical discharge machining parameters using artificia neural network with different electrodes" 5th Inter- national \& 26th All India Manufacturing Technology, Design and Research Conference (AIMTDR 2014), IIT Guwahati, Assam, India, (2014).

[12] Kapil Banker, Ujjval Prajapati, Jaimin Prajapati, Paras Modi "Parameter optimization of Electro Discharge Machine of AISI 304 Steel by using Taguchi Method" International Journal of Application or Innovation in Engineering \& Man- agement (IJAIEM)(2014)

[13] Dr. M.Indira Rani, Ketan "Optimization of Various Machin- ing Parameters of Electrical Discharge Machining (EDM) Process on AIS D2 Tool Steel Using Hybrid Optimization Method" International Journal of Application or Innovation in Engineering \& Management (IJAIEM) (2014).

[14] Chandramouli S, Shrinivas Balraj U and Eswaraiah K“Optimization of Electrical Discharge Machining Process Parameters Using Taguchi Method" International Journal of Engineering Research and Applications (IJERA), (2014).

[15] Raghuraman S, Thiruppathi K, Panneerselvam T, Santosh S, "Optimization of edm parameters using taguchi method and grey relational analysis for mild steel is 2026", Journal of In- novative Research in Science, Engineering and Technology, (2013).

[16] M.A. Ali, M. Samsul, N.I.S. Hussein, M. Rizal, R. Izamshah, M. Hadzley, M.S. Kasim, M.A. Sulaiman and S. Sivarao, "The effect of edm die-sinking parameters on material re- moval rate of beryllium copper using full factorial method", Journal of Scientific Research, (2013).

[17] S. Assarzadeh, M. Ghoreishi, "Statistical modeling and opti- mization of process parameters in electro-discharge machin- ing of cobalt-bonded tungsten carbide composite (WC/6\%Co)", The Seventeenth CIRP Conference on Electro Physical and Chemical Machining (ISEM), (2013).

[18] M. Gostimirovic, p. Kovac, B. Skoric, M Sekulic, "Effect of electrical pulse parameters on the machining performance in EDM", Indian Journal of engineering and materials sciences, (2012).

[19] Ramezan Ali MahdaviNejad, "Modeling and optimization of electrical discharge machining of sic parameters, using neural network and non-dominating sorting genetic algorithm (NSGA II)", Journal of Scientific Research, Materials Sci- ences and Applications, (2011).

[20] P. Kuppan, A. Rajadurai, S. Narayanan, "Influence of EDM process parameters in deep hole drilling of Inconel 718", Journal Advance Manufacturing Technology, (2008).

[21] M. Kiyak, O. Cakir, "Examination of machining parameters on surface roughness in EDM of Tool Steel", Journal of Ma- terials Processing Technology, (2007).

[22] Kun Ling Wu, Biing Hwa Yan, Fuang Yuan Huang, Shin Chang Chen, "Improvement of surface finish on SKD steel using electrodischarge machining with aluminum and sur- factant added dielectric", Journal of Machine Tools \& Manu- facture (2005). 